\title{
Editorial
}

\section{The Quality of Rehabilitation}

Quality, quality circles, quality assurance, quality control managers are all words heard more and more frequently by health care workers today. Quality in medicine has been pursued since Hippocrates, and life as opposed to death has always been the crude measure of success. In these days of more sophisticated and increasingly expensive medical care, finer measures of its degree or grade of excellence are obviously required.

Economic pressures are forcing health care workers to look at their efficiency and effectiveness, and whilst all would agree that this is important, many are concerned that the results reflect quantity at the expense of quality.

Measurement of the quality of rehabilitation is particularly complex because of the contribution of so many people. It would appear that most professional people, irrespective of their experience, have an innate sense of what constitutes quality for their area of work. The difficulty lies not in its recognition, but in its measurement. Few tools for measuring rehabilitation have been developed and even those such as psychological tests and indices for the activities of daily living, are not widely used or agreed!

For patients with trauma to the spinal cord, rehabilitation is affected from the outset by the actions of ambulance crews or others involved in giving the first aid. Rehabilitation in hospital prepares the patient to function in the community but it is also essential to prepare the community to minimise the handicap of the disabled person. Preparation of the environment calls for the knowledge and skills of people such as architects, city planners and designers of transport, far removed from the clinical scenario of early rehabilitation activities.

Until now, hospital care has received the most attention for without 'good' initial care people would not live or be able to move back into their own homes and communities. How much of the effort of the hospital team is rendered ineffective by poor provision in the community?

What is deemed to be high quality or even acceptable care for those with spinal lesions? How is it measured? Peer review audit is considered a useful tool in studying the outcome of clinical practice of all those involved. In assessing the quality of care, the organisation and management of the services to patients are also important and in some countries data on these aspects form the basis for enforced measures to monitor quality. What has been the contribution of these accreditation systems to the care of patients with spinal cord injuries in such countries?

How is progress in the measurement of quality to be made? Should minimal acceptable standards be set and enforced by an outside agency? Is it too idealistic to rely upon individual enthusiasm to improve patient care? How are we to resolve the problem surrounding the measurement of all aspects of the rehabilitation of patients with spinal cord lesions? Perhaps Paraplegia could act as a forum for the exchange of ideas on this important matter? 